\title{
Tuning Optimization Algorithms for Real-World Problems by Means of Surrogate Modeling
}

\author{
Mike Preuss \\ Günter Rudolph \\ Simon Wessing \\ Computational Intelligence Group, Chair of Algorithm Engineering \\ Technische Universität Dortmund, Germany \\ \{mike.preuss,guenter.rudolph,simon.wessing\}@tu-dortmund.de
}

\begin{abstract}
The case-specific tuning of parameters of optimization metaheuristics like evolutionary algorithms almost always leads to significant improvements in performance. But if the evaluation of the objective function is computationally expensive, which is typically the case for real-worlds problems, an extensive parameter tuning phase on the original problem is prohibitive. Therefore we have developed another approach: Provided that a (computationally cheap) surrogate model is available that reflects the structural characteristics of the original problem then the parameter tuning can be run on the surrogate problem before using the best parameters thereby identified for the metaheuristic when optimizing the original problem.

In this experimental study we aim to assess how many function evaluations on the original problem are necessary to build a surrogate model endowed with the characteristics of the original problem and to develop a methodology that measures to which extent such a matching has been achieved.
\end{abstract}

\section{Categories and Subject Descriptors}

G.3 [Probabilistic Algorithm]: Evolutionary Algorithm; G.1.6 [Optimization]: Global Optimization-computationally expensive objective function, real-world problem; G.1.2 [Approximation]: Nonlinear Approximation-metamodel, surrogate model, kriging; I.2.6 [Learning]: Parameter Learning - parameter tuning

\section{General Terms}

algorithms, experimentation

\section{Keywords}

parameter tuning, surrogate model, experimental study

Permission to make digital or hard copies of all or part of this work for personal or classroom use is granted without fee provided that copies are not made or distributed for profit or commercial advantage and that copies bear this notice and the full citation on the first page. To copy otherwise, to republish, to post on servers or to redistribute to lists, requires prior specific permission and/or a fee.

GECCO'10, July 7-11, 2010, Portland, Oregon, USA.

Copyright 2010 ACM 978-1-4503-0072-8/10/07 ...\$10.00.

\section{INTRODUCTION}

Tuning is gaining ground in Evolutionary Computation as it has been realized that setting parameters of an optimization algorithm right can lead to enormous performance gains. However, one of the yet unsolved problems is how to apply tuning in a real-world setting, where evaluations are costly. Tuning an optimization algorithm on the original problem is clearly infeasible. Thus, one is left with the default parameters or modifications on these one can justify by problem knowledge and comparison to related problems. Alternatively, one could think of several default parameter settings. E.g., it is well known that the CMA-ES [9] which is currently regarded as the evolutionary standard algorithm for real-valued problems, does not cope very well with the Rastrigin problem if its population is not artificially enlarged [11]. This led to the development of a deterministic population size increase with every restart as suggested in $[1]$.

There is no doubt that the resulting algorithm is more robust than the former variant without population enlargement. However, one could argue that it uses an implicit, heuristic tuning rule: Once the CMA-ES reaches a stagnation phase without attaining the targeted quality, it is presumed that a) the tackled problem is multimodal so that the run went into the wrong basin, and that b) this can be cured by restarting with enlarged population, possibly exploiting a strong global structure that becomes visible to the algorithm if enough information gets available to 'average out' the misleading local optima. However, a deterministic increase would be rather harmful on problems without such strong global structure where it may be much better to do restarts with small populations. But how could a decision in favor of or against enlarged population be made without investing the evaluations for several restarts?

Another parameter that frequently desires some attention when tackling an unknown problem by means of an EA is the initial step size. Since the invention of self-adaptation (see [4] for a comprehensive introduction), evolutionary algorithms usually come with some means of learning a suitable step size. So also does the CMA-ES. In either case, some function evaluations have to be sacrificed for getting into the range of high performance if the default step size was too large or small. This is not a problem if evaluations are cheap. However, if they are costly, optimization runs are naturally bounded in length and the available time may not suffice for adjusting all parameters and then apply them. It shall be noted that the aforementioned parameter setting problems 
are found within all EAs, often in much more demanding form than for the CMA-ES.

In this work we suggest an approach to tune an EA by means of a surrogate model of the problem itself. This is fundamentally different from applying meta-model supported tuning methods as, e.g., the sequential parameter optimization SPO [2]. However, application area and desired performance gain are also quite dissimilar. Our setting is the one of very costly function evaluations, and we desire to adjust the parameters of a given optimization algorithm not to maximal performance, but rather want to get into the 'ball park'. This term has been used by de Jong [12] to express that it is usually much easier to attain reasonable performance settings instead of the best possible ones.

Note that our approach is also different from integrating a surrogate model with the optimization algorithm directly as suggested, e.g., by Emmerich et al. [6] and Hansen et al. [13]. These utilize the function values already obtained during the optimization run to predict areas with high probability of improvement. We argue that for tuning, different properties of the model are used so that from success or failure of either approach it is not possible to conclude on the other.

In the following, we elaborate on the envisioned tuning method and shortly recite the first previously reported use case. Whether the general suitability of the method has already been documented $[16]^{1}$, the overall aim of this work is to show that the approach works well enough to allow for improved parameter settings while using only small samples of the original problem. We then apply our approach to compare CMA-ES parameter effects on two well known test functions and their surrogate models.

\section{TUNING VIA SURROGATE MODEL OF THE OPTIMIZATION PROBLEM}

The general idea of our approach is to use few samples of an optimization problem gathered via test runs to establish a surrogate model and then to tune the chosen algorithm on this model. If the model honors the actually observed values, it is clear that for the number of samples approaching infinity, there must be some point where the model is so close to the original problem that they become indistinguishable from the viewpoint of the optimization algorithm. For this reason, we employ a Kriging model as default.

\subsection{Kriging Surrogate Model}

This model originates from geostatistics and has been introduced into optimization by Sacks et al. [17]. Assuming that no knowledge concerning the global structure of the problem is at hand, it shall be safe to rely on ordinary Kriging which abstains from any polynomial regression component. For the experiments below, we employ a Python reimplementation of the DACE Matlab toolbox [14]. A beginnerfriendly introduction to Kriging can be found in Forrester et al. [7], while Santner et al. [18] discuss the topic in great detail. Formally, our task is to build a surrogate model from a number of points $\mathbf{X}=\left\{\mathbf{x}^{(1)}, \mathbf{x}^{(2)}, \ldots, \mathbf{x}^{(n)}\right\}^{\mathrm{T}}$ in $k$ dimensions and associated responses $\mathbf{y}=\left\{y^{(1)}, y^{(2)}, \ldots, y^{(n)}\right\}^{\mathrm{T}}$. Our assumption now is that the $y^{(i)}$ are realizations drawn from a stochastic process $\mathbf{Y}(\cdot)$. To model the interpolating

\footnotetext{
$\overline{{ }^{1} \text { presented at ENBIS/EMSE-2009 }}$
}

response surface, Kriging uses correlation functions

$$
\operatorname{cor}\left[Y\left(\mathbf{x}^{(i)}\right), Y\left(\mathbf{x}^{(l)}\right)\right]=\exp \left(-\sum_{j=1}^{k} \theta_{j}\left|x_{j}^{(i)}-x_{j}^{(l)}\right|^{p_{j}}\right)
$$

as a kernel. In our case, $p_{j}=2$ is kept fixed, representing a gaussian kernel. The $\theta_{j}$ are controlling the 'smoothness' of the model. For example, a low $\theta_{j}$ means that sampled points influence a wide-spread area in dimension $j$. So, finding the optimal $\boldsymbol{\theta}$ is unfortunately itself an optimization problem, embedded in the model-fitting process. Usually, the likelihood of obtaining $\mathbf{y}$ from the given model is used as objective function in this case. Lophaven et al. [14] employ a customized pattern search method called boxmin to tackle the problem. Wagner et al. [5] instead use the already mentioned CMA-ES [9], but do not compare with the boxmin performance.

Note that the literature on statistical models is rich, so that Kriging models are by no means the only possibility to try. However, we choose it for two reasons: a) we need to make only very rough assumptions concerning the form of the modeled problem, and b) Kriging is a local model that weights neighboring points much higher than distant points. It is thus possible to represent heterogeneous areas of the search space with a single model. Kriging's feature of estimating the prediction uncertainty is not employed here.

\subsection{Problem Properties}

Nevertheless, it is not clear which features of the underlying problem the model must contain to facilitate tuning. Certainly, accuracy is much less important than topology. Any invariance property of the optimization algorithm can be safely disregarded for modeling, as e.g. scaling or rotation of the target function for the CMA-ES. The parameters of evolution strategies (ES) should not depend on the concrete target function values but rather on their ranks as the ES selection operator is rank-based. But which are the important problem properties?

One reason for the current attention to tuning methods stems from the fact that we currently do not possess a good understanding of the interplay of algorithm parameters and problem properties. Otherwise, it would be possible to construct a good parameter set at least for any well-known problem. Experience provides us with some rules of thumb concerning the parameter/problem matching, but they are far from reliable. We may partition the problem properties into the ones relating to global structure and the ones relating to local convergence. For the sake of simplicity, we may assume that our optimization algorithm is able to deal well with the second group, so that our model only has to capture the first group.

Some global structure related problem properties which may induce a parameter effect are (also compare [20], [8], and [3]):

- degree of modality: Number of local optima

- strong/weak global basin structure

- size distribution of the basins of attraction

- search space distribution of the basins

- existence of neutral search space parts (plateaus)

However, this list is neither complete and nor is it trivial to assign concrete values to the properties. Note that the number of local optima could be estimated even for an unknown function, but this is only possible by doing many 
Table 1: CMA-ES parameter intervals and grid sample values for tuning and model check

$\begin{array}{lllll}\text { CMA-ES } & \text { initial stepsize } & \text { parent population size }(\mu) & \text { damp multiplier }\left(x \cdot d_{\sigma}\right) & \text { restart base } \\ \text { Domains } & 0.004: 2.048 & 2: 50 & 0.2 \cdot \sqrt{2}^{m-1} & 1.0+0.2 \cdot m-1 \\ \text { Values } & 0.004 \cdot 2^{m-1} & 2,3,4,5,7,10,15,20,30,50 & 0.2: 4.6 & 1.0: 2.8 \\ \text { Default 10D } & 1 & 5 & 1 & 2 \\ \text { Default 2D } & 1 & 3 & 1 & 2\end{array}$

local search runs which is infeasible under tight running time restrictions.

\section{EXPERIMENTAL INVESTIGATION}

In all our following experiments, we assume that we possess a tuning method that detects good parameter settings when they exist. We thus concentrate on investigating parameter induced algorithm behavior similarities between runs on the original problem and runs on a model. But how to compare performance data between these two?

Of the many possibilities, we choose a large $\left(10^{4}\right.$, according to 10 levels of 4 parameters, see sect. 3.2) grid design over the parameter space. Assuming a certain smoothness in the performance-parameter relationship, we expect to be at least in the range of the best and worst performance possible. Applying a tuning method may of course reveal even better parameter sets, but either the difference is not large or the obtained parameters sets are very sensible, which is undesired.

\subsection{Stopping Criteria}

There are two principal ways to stop a restart-enabled optimization algorithm: Either a certain target function value shall be reached (called 'horizontal' measuring by Hansen et al. [8]), or we run until a predefined function evaluation bound is hit ('vertical' in [8]). Although we support the view that going for the time needed to attain the required accuracy is often the preferable form of measuring, we use different terms in the following, hoping that they are less confusing. We call stopping when achieving a predefined accuracy the 'quality-task' criterion, if running up to a tightly bound number of evaluations the 'budget-task' criterion. For optimization runs on the surrogate model, a predefined quality is not available as the best values predicted may be above or even below the global optimum. We therefore need to employ the budget-task criterion.

\subsection{CMA-ES Parameters}

As previously stated, our optimization algorithm of choice is the CMA-ES, which comes with a robust rule set for obtaining default parameters, depending on the number of search space dimensions. These parameters usually lead to good performance but still bear some potential for speedups. Here, we employ a subset of the parameters tested in [15], restricted to the seemingly most relevant ones: Population size $\lambda$ (we use $\mu$ just for convenience, with $2 \mu=\lambda$ ), initial step size $\sigma$, damping factor for step size adaptation $d_{\sigma}$, and the base factor for incrementing population size. This last parameter is usually set to 2 and would lead to slower or faster population growth for values below 2 or higher than 2 , respectively. CMA-ES parameter ranges and default values are given in table 1 .

For each of the 4 varied parameters, 10 values are spread over the ranges, mostly with a logarithmic scaling. This follows from a first parameter sensitivity check where a linear scaling resulted in a too coarse grid for the small parameter values.

Note that in the default parameters, we set the intial step size to 1 for the Rastrigin and the Schwefel 2.13 problem. This is slightly smaller than recommended in [10] (where $0.3 \cdot(b-a)$ is used) and translates to 3 for Rastrigin and $0.6 \pi \approx 1.89$, however this should rather be an advantage than a disadvantage on highly multimodal functions.

\subsection{Measures: TQP and TQM}

In order to assess to which extent the surrogate model reflects the characteristics of the original problem we need an appropriate scalar measure. We assume that we have an appropriate approximation if the $25 \%$ of the best parameterizations predicted from the surrogate model are also among the $25 \%$ of the best parametrizations identified for the original problem. Therefore we count how many of the $25 \%$ best parametrizations predicted from the surrogate model are actually in the set of $25 \%$ best parameterizations for the original problem. These parameterizations may be interpreted as "true positives". Accordingly, we can define "false positives", i.e., where a parameterization predicted among the $25 \%$ best is actually worse than the median parametrization for the true problem.

A formal definition of these measures requires some preparations: Let $N$ be the number of different parameterizations $\omega_{1}, \ldots, \omega_{N}$ to be set for some algorithm $A$. Suppose there exists a performance measure that assigns a real value to each possible output of some algorithm such that better outputs get larger values. Let $m_{i}=A_{f}\left(\omega_{i}, r\right)$ denote the median performance from $r$ runs of algorithm $A$ applied to objective function $f(\cdot)$ with parameterization $\omega_{i}$. Similarly, $\tilde{m}_{i}=A_{\tilde{f}}\left(\omega_{i}, r\right)$ where $\tilde{f}$ stands for the surrogate model of the original function $f$. Arrange the pairs $\left(m_{i}, \omega_{i}\right)$ such that the $m_{i}$ appear in ascending order. Select the first quarter of the pairs, extract the parameterizations $\omega_{i}$ from the pairs and gather them in the set $Q_{1}$. Select the second (third, fourth) quarter of the pairs and store the extracted parameterizations $\omega_{i}$ in the set $Q_{2}\left(Q_{3}, Q_{4}\right)$. Do the same for the pairs $\left(\tilde{m}_{i}, \omega_{i}\right)$ yielding the sets $\tilde{Q}_{1}, \ldots, \tilde{Q}_{4}$.

Definition 1 Let $Q_{1}, \ldots, Q_{4}$ be the quarters of the ordered parameterizations for the original problem and $\tilde{Q}_{1}, \ldots, \tilde{Q}_{4}$ for the surrogate problem. The quantity

$$
\mathrm{TQP}=\frac{1}{\left|\tilde{Q}_{4}\right|} \sum_{\omega \in \tilde{Q}_{4}} 1_{Q_{4}}(\omega)
$$

is termed the top quarter prediction whereas the quantity

$$
\mathrm{TQM}=\frac{1}{\left|\tilde{Q}_{4}\right|} \sum_{\omega \in \tilde{Q}_{4}} 1_{Q_{1} \cup Q_{2}}(\omega)
$$

is called the top quarter misprediction. 

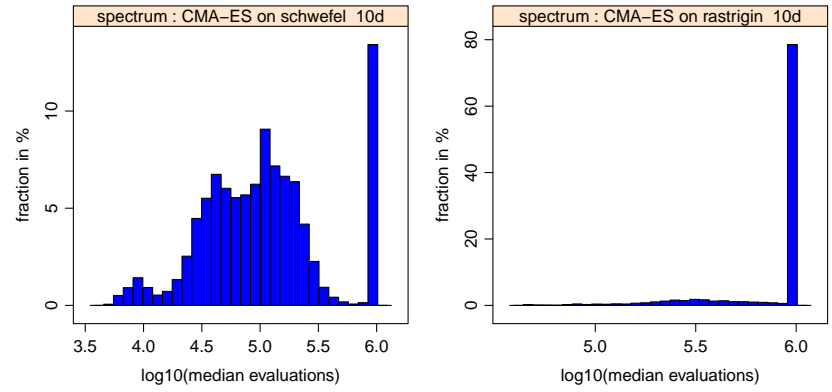

Figure 1: Histogram of quality-task grid sample of 10D Schwefel 2.13 (left) and Rastrigin function (right) with runtime limit $10^{6}$ evaluations

\subsection{Test Problems}

As primary test problems, we choose F10 and F12 from the CEC'05 benchmark set [19]. Note that for the CMA$\mathrm{ES}$, the two Rastrigin functions of the set F9 (unrotated) and F10 (rotated) should be similar in performances due to the algorithm's invariance against rotation and shifting. F12 is also known as problem Schwefel 2.13. The choice of these problems is motivated by the need for one representative of a strong global structure (Rastrigin) and one of a weak global structure (Schwefel). Both problems are highly multimodal. We test in 10 and 2 dimensions.

\subsection{Schwefel 2.13 and Rastrigin, 10D}

We start the experimental investigation with the attempt to tune the CMA-ES on models of different quality of two standard benchmark functions.

Experiment: Is a small Kriging model sufficient for representing standard benchmark functions?

Pre-experimental planning. At first, we need to detect suitable runtimes to ensure that there is some spread in the performance over the different parameter sets on the original problems. Figure 1 shows the histograms of all $10^{4}$ grid samples, with 21 repeats each. The runs were terminated by the quality-task criterion when approaching the global optimum with an error of less than 0.01 or when hitting the maximal number of function evaluations which was set to $10^{6}$. We note that the spread is much higher for the Schwefel 2.13 function, while only few configurations ensure good performance for the Rastrigin function. The best parameter sets enable reaching the target in $\approx 5000$ and $\approx 50000$ function evaluations for Schwefel 2.13 and Rastrigin, respectively.

Task. We test the hypothesis that the original problems can be approximated accurately enough by our model to allow for tuning. Therefore, the TQP should be at least in the range of $50 \%$.

Setup. The CMA-ES is run in a $10^{4}$ grid sample (see table 1) on Kriging models of Schwefel 2.13 and Rastrigin function made of 100, 200 and 400 randomly sampled points, with 5 repeats (the number of repeats is reduced as runs on the model are considerably slower than on the original function). Additionally, the same sample is done on the original functions with a budget-task of $10^{4}$ and $10^{5}$ for the Schwefel 2.13 and the Rastrigin function, respectively. The models are build without employing an additional embedded optimization of the $\theta$-values.
Table 2: Correlations between grid samples on Schwefel 2.13 original function and on Kriging models. Quality means quality-task, budget stands for budget-task and $\mathrm{m}-\mathrm{X}$ refers to the model with $\mathrm{X}$ as random samples used for creating the model.

\begin{tabular}{|rrrrrr|}
\hline & quality & budget & $\mathrm{m}-100$ & $\mathrm{~m}-200$ & $\mathrm{~m}-400$ \\
quality & 1.000 & 0.036 & 0.189 & 0.187 & 0.194 \\
budget & 0.036 & 1.000 & 0.033 & 0.041 & 0.031 \\
m-100 & 0.189 & 0.033 & 1.000 & 0.529 & 0.528 \\
$\mathrm{~m}-200$ & 0.187 & 0.041 & 0.529 & 1.000 & 0.524 \\
$\mathrm{~m}-400$ & 0.194 & 0.031 & 0.528 & 0.524 & 1.000
\end{tabular}

We measure Kendall's $\tau$ rank correlation between the median values of all 5 samples per function (including the qualitytask runs of the pre-experimental planning), and compute TQP and TQM between the model-based results and the original function results.

Results/Visualization. For the Schwefel 2.13 function, table 2 reports the measured correlations, and table 3 the resulting TQP and TQM measures. For the Rastrigin function, the experiment has been cancelled after obtaining the results for the model with 100 points. The measured TQP for concluding from the model to the quality-task performance rank is far below 0.4. A severe problem with the model was deduced which led to the experiment described in section 3.6.

Observations. As stated, the measured TQP values for the Rastrigin function are suprisingly bad and do not allow for a good prediction of the parameter set rank. For Schwefel 2.13, the rank correlations between the models and the quality-task are also suprisingly small, and seem to improve rather slowly with increasing number of points. The TQP measures show a slight trend of improvement for the qualitytask, with relatively low TQM values. For the budget-task, the TQP measures are far below usability, and the TQM values extremely high.

Discussion. Given that a complete random order of parameter sets would achieve a TQP measure of $25 \%$, the modelbased quality prediction obviously failed for the Rastrigin function. For the Schwefel 2.13 function, we get at least an accuracy of around $45 \%$ for concluding from the rough performance rank on the model to the rough performance rank on the original function in case of the quality-task. Here, the model failed to achieve the task of $50 \%$ only by a small margin. Test runs with a higher number of repeats (21) indicate that the TQP improves to the range of $50 \%$ by utilizing more accurate data. This points to a considerable amount of variation in the data which is used for establishing the rankings. For the budget-task, all models behave so different from the original function that no predictions are

Table 3: TQP and TQM performance rank prediction measures for concluding from the model-based results to the original Schwefel 2.13 function results

\begin{tabular}{|rcccccc|} 
from & \multicolumn{2}{c}{$1 \mathrm{~m}-100$} & \multicolumn{2}{c}{$1 \mathrm{~m}-200$} & \multicolumn{2}{c|}{$1 \mathrm{~m}-400$} \\
to & TQP & TQM & TQP & TQM & TQP & TQM \\
\hline quality & 0.451 & 0.239 & 0.457 & 0.224 & 0.458 & 0.227 \\
budget & 0.091 & 0.617 & 0.091 & 0.603 & 0.099 & 0.607
\end{tabular}


Table 4: Number of successful modelings of the original Rastrigin function.

\begin{tabular}{|rrrrr|}
\hline & \multicolumn{3}{c}{ 1D } & \multicolumn{2}{c|}{ Points } & CMA-ES & boxmin & CMA-ES & boxmin \\
25 & 3 & 0 & 0 & 0 \\
50 & 4 & 0 & 0 & 0 \\
100 & 3 & 0 & 0 & 0 \\
200 & 5 & 0 & 5 & 1 \\
400 & 5 & 0 & 5 & 0 \\
800 & 5 & 5 & 5 & 0
\end{tabular}

Table 5: Number of successful modelings of the CEC2005 Rastrigin function $\left(F_{9}\right)$.

\begin{tabular}{|rrrrr|} 
& \multicolumn{3}{c}{${ }^{1 D}$} & \\
Points & CMA-ES & boxmin & CMA-ES & boxmin \\
25 & 3 & 0 & 0 & 0 \\
50 & 5 & 0 & 0 & 0 \\
100 & 3 & 0 & 0 & 0 \\
200 & 5 & 0 & 5 & 2 \\
400 & 5 & 5 & 5 & 0 \\
800 & 5 & 5 & 5 & 0
\end{tabular}

possible, looking for top-performance parameter sets on the model would even lead to very bad performing parameter sets on the original function. Overall, we may assume that there is some potential in the approach but that the utilized test functions are on the border of what the models can manage or beyond. We need to search for ways to improve the model as the number of points cannot be increased by a large margin in the context of real-world applications.

\subsection{Assessing and Improving Model Quality}

Experiment: Under which conditions does model quality break down and can this be cured by optimizing $\boldsymbol{\theta}$ ?

Pre-experimental planning. Experiment 3.5 showed that our approach did not work as expected on the Rastrigin function. When tracking down the root of the problem, we found out that the Rastrigin function was quite difficult to model. Surprisingly, the Kriging model even showed some regression behavior, which contradicts its theoretically guaranteed feature of interpolating all support points.

Task. The experiment should help determining conditions and situations which prevent or allow modelling of the optimization problem.

Setup. To get a broader perspective, we add two related variants of the Schwefel and Rastrigin function. The sinesqrt-Schwefel function reads

$$
f(\mathbf{x})=418.9829 \cdot k+\sum_{i=1}^{k}-x_{i} \cdot \sin \left(\sqrt{\left|x_{i}\right|}\right)
$$

and is defined on $-500 \leq x_{i} \leq 500$. To visualize the Kriging models, Schwefel, Rastrigin, and their two modifications from CEC $2005\left(F_{9}\right.$ and $\left.F_{12}\right)$ are examined in 1D and 2D only. On each problem, models are created with six different sample sizes. Each sample is chosen with a latin hypercube design. For each sample we fit one model with CMA-ES and one with boxmin. Both algorithms have internal stopping criteria, so we leave the budget of maximum likelihood esti-
Table 6: Number of successful modelings of the Sinesqrt Schwefel function.

\begin{tabular}{|rrrrr|} 
& \multicolumn{3}{c}{${ }^{2 \mathrm{D}}$} & \\
Points & CMA-ES & boxmin & CMA-ES & boxmin \\
25 & 3 & 0 & 0 & 0 \\
50 & 3 & 5 & 0 & 0 \\
100 & 5 & 5 & 5 & 5 \\
200 & 5 & 5 & 5 & 3 \\
400 & 5 & 5 & 5 & 0 \\
800 & 5 & 5 & 5 & 5
\end{tabular}

mations unlimited. However, the algorithms were designed with very different intentions. CMA-ES runs until convergence is reached, while boxmin's aim is to keep the fitting time as low as possible. In effect, boxmin seldom makes more than 15 evaluations, while CMA-ES easily tops that by a factor of 100. This budget is similar to that of Wagner et al. [5], who allowed 2000 evaluations per dimension. We make five repeats per sample size to get a basic understanding of random effects. Furthermore we restrict the search space for $\boldsymbol{\theta}$ by requiring $0.01 \leq \theta_{j} \leq 100$.

Results/Visualization. Tables 4, 5, and 6 contain information on how often each Kriging variant could model the original problem. There is no table for $F_{12}$ because it could be always modeled successfully. Figure 2 shows some interesting effects on one-dimensional problems. Similarly, Figure 3 illustrates how different the results on two-dimensional problems are.

Observations. Figure 3 shows that the problems have different levels of difficulty. When too few points are used to build the model, undesirable effects like in Figure 3(a) can appear. There, the extensive optimization with CMA-ES leads to a distorted model, finding only the structure in one dimension. The classical boxmin cannot identify any structures either and sticks with a few local bumps. Figures 3(b) and $3(\mathrm{c})$ show that boxmin tends to surfaces that are too smooth.

Discussion. The tables and figures show that Kriging with boxmin is able to model the one-dimensional functions if the sample size is sufficiently high. However, this means in many cases disproportionately high numbers. The results for the CMA-ES variant are much more convenient. The biggest difference can be found on two-dimensional Rastrigin problems, where boxmin Kriging never leaves the regression mode.

It is reasonable to invest into the model's expensive optimization in our application, because the model only needs to be fitted once. After that, the optimization algorithm can be tuned on the model. Therefore, the model optimization may well take a few minutes. But we also saw that Kriging's capabilities are not unlimited and we still have no general rule to predict if a function can be modeled.

\subsection{Rastrigin 2D with Embedded CMA-ES}

In contrast to experiment 3.5, we now utilize an embedded CMA-ES for optimizing the $\theta$-values of the Kriging model. We focus on the Rastrigin function as it has been especially tough to model without embedded optimization and reduce the dimensionality to 2 . Figure 3 indicates that with embedded $\theta$-value optimization, models from about 100 base 


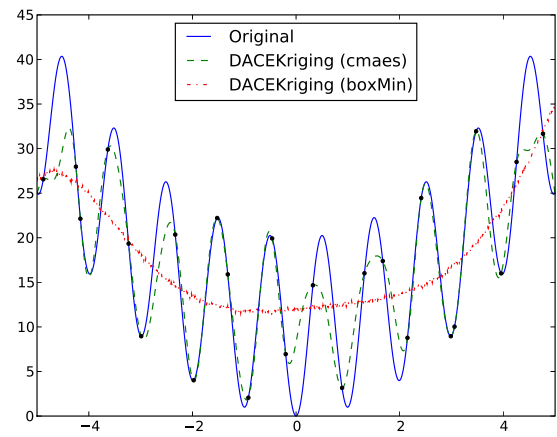

(a) Rastrigin, 25 points

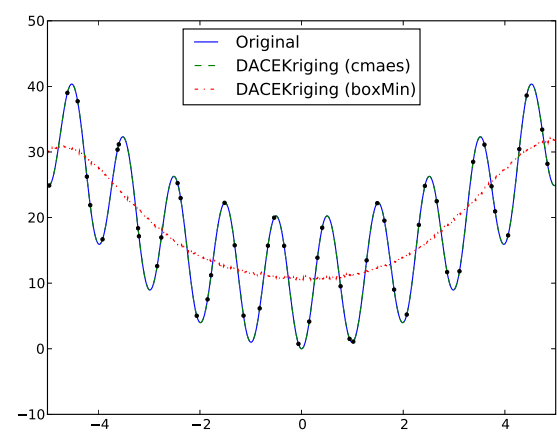

(b) Rastrigin, 50 points

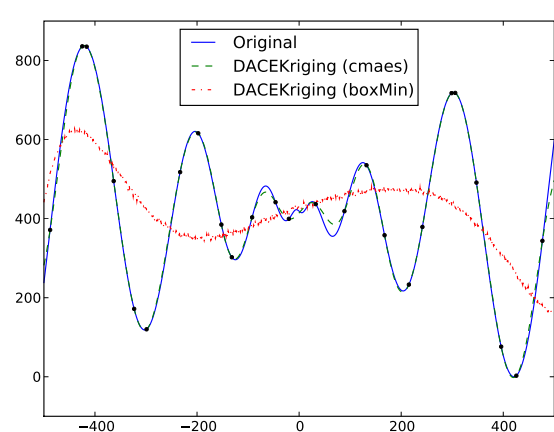

(c) Sine-sqrt Schwefel, 25 points

Figure 2: Different Kriging models built from the Rastrigin and Schwefel functions. Boxmin clearly fails to interpolate the points

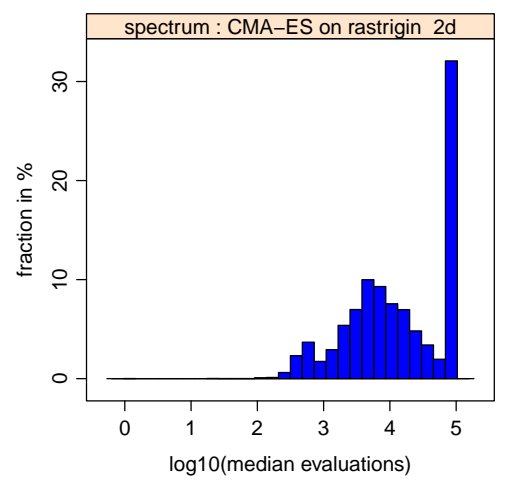

Figure 4: Histogram of quality-task grid sample of 2D Rastrigin function with runtime limit $10^{5}$ evaluations

points upwards shall be good enough to capture the main characteristics of the problem.

Experiment: Does the embedded optimization improve the model fit sufficiently for making rough rank predictions?

Pre-experimental planning. As for our first experiment, we need to determine suitable run times for the available parameterizations on chosen function (Rastrigin 2D). Figure 4 shows the histogram of all $10^{4}$ grid samples, with 21 repeats each. The best parameterizations lead to runtimes of less than 1000 evaluations and the spread is remarkably high.

Task. Our hypothesis is that the improved model generation allows for TQP values of around $50 \%$.

Setup. The setup largely resembles the one for experiment 3.5, with the following modifications: We only test on the 2D Rastrigin function, the budget for the runs on the model is set to 5000, and we employ the CMA-ES as embed$\operatorname{ded} \theta$-value optimization while establishing the model, with 1000 evaluations (of $\boldsymbol{\theta}$ ) allowed. Models with 100, 200, and 400 points are tried.

Results/Visualization. Table 7 shows the rank correlations between parameter set measurements on the original function and the models, table 8 the resulting TQP and TQM measures. Figure 5 shows the original function and the model obtained with 100 points.
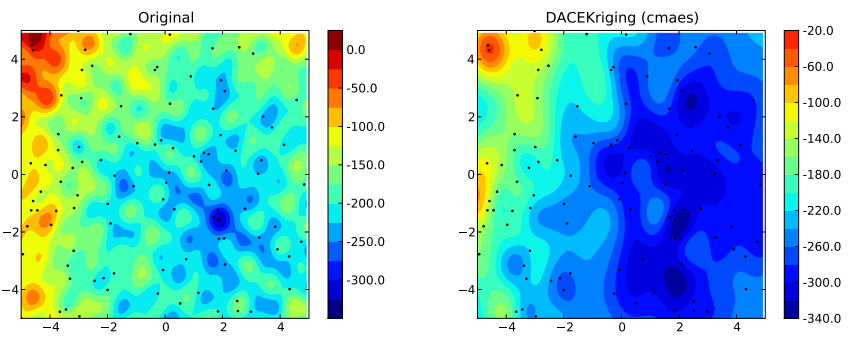

Figure 5: Rastrigin (F10) 2D test function (left) and model derived from 100 random samples (right)

Observations. The reported rank correlations are remarkably high, although direct comparison to the values of table 2 is not possible (different problem). However, it is strange to see that the correlations decrease with larger point numbers, whether the TQP measures increase as desired. Figure 5 shows that the main characteristics of the problem are captured well, although the basin structure representation could be improved. The achieved TQP values are slightly less than demanded, but the TQM values are also well below $50 \%$.

Discussion. The 100-point model is already useful for tuning as documented by the TQP and TQM values. They mean that taking a 'good' setting (top quarter) from model tuning gets us a below median parameterization for the real problem with low probability (TQM). We miss the TQP target of $50 \%$ by some margin, but already get near to it with the 200 and 400 point models. Although we already use 21 repeats, there is still some noise in the rankings so that the

Table 7: Rank correlations between grid samples on Rastrigin 2D function and on Kriging models. Symbol meanings as in table 2

$\begin{array}{rrrrrr} & \text { quality } & \text { budget } & \mathrm{m}-100 & \mathrm{~m}-200 & \mathrm{~m}-400 \\ \text { quality } & 1.000 & 0.640 & 0.349 & 0.377 & 0.371 \\ \text { budget } & 0.640 & 1.000 & 0.293 & 0.293 & 0.282 \\ \text { m-100 } & 0.349 & 0.293 & 1.000 & 0.637 & 0.651 \\ \mathrm{~m}-200 & 0.376 & 0.294 & 0.637 & 1.000 & 0.620 \\ \mathrm{~m}-400 & 0.371 & 0.282 & 0.651 & 0.620 & 1.000\end{array}$



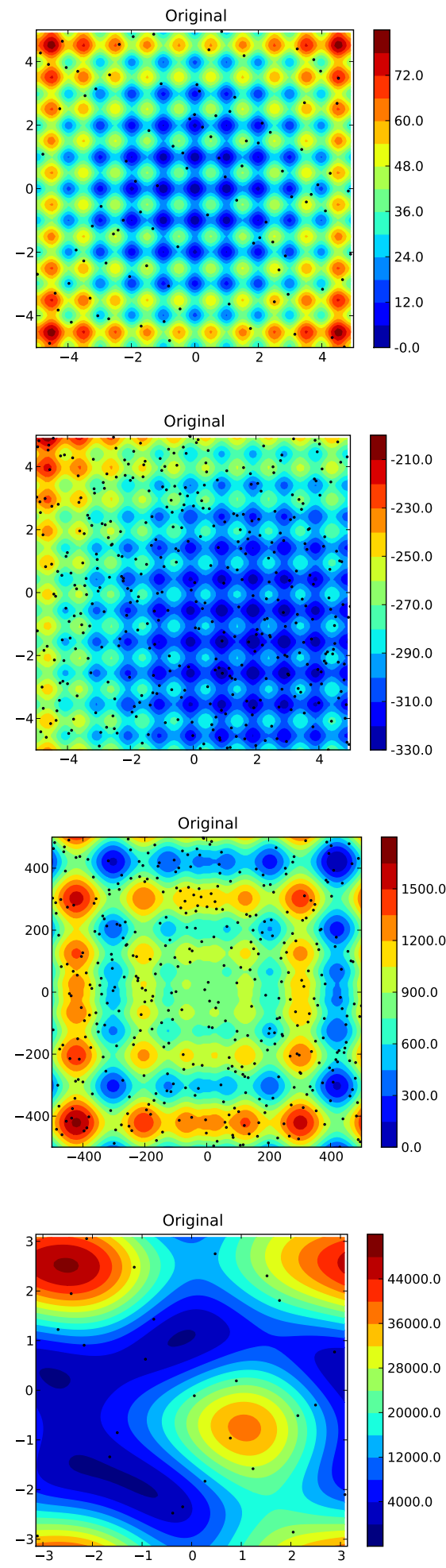

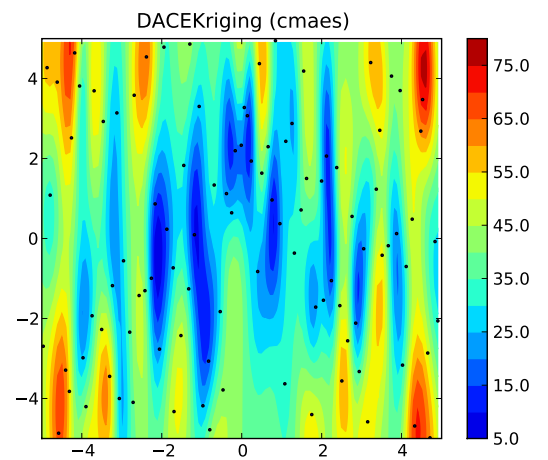

(a) Rastrigin with 100 points.

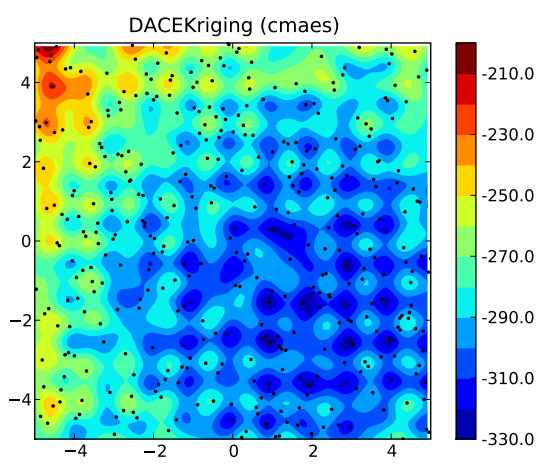

(b) CEC 2005 Rastrigin $\left(F_{9}\right)$ with 400 points.

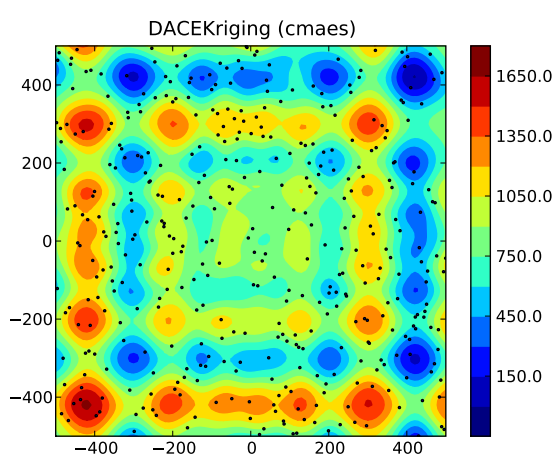

(c) Sine-sqrt Schwefel with 400 points.

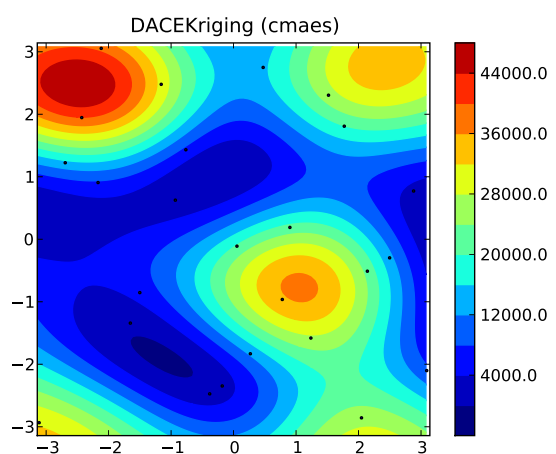

(d) CEC 2005 Schwefel $\left(F_{12}\right)$ with 25 points
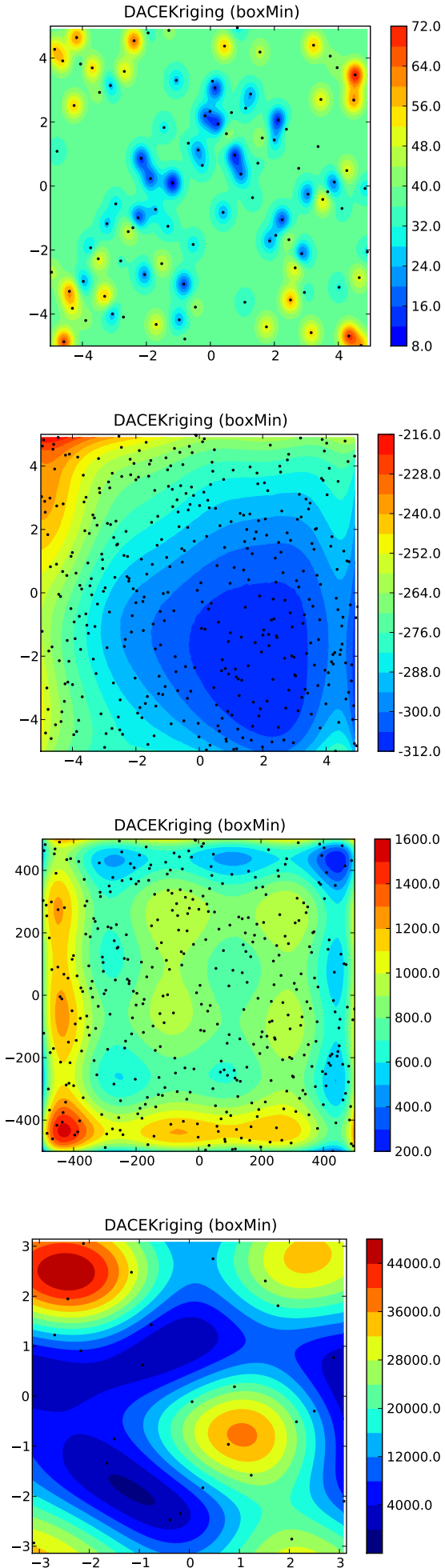

Figure 3: Original function plots (left) and Kriging models for different two-dimensional problems. Middle column: CMA-ES embedded optmization, right column: boxMin embedded optimization 
Table 8: TQP and TQM performance rank prediction measures for concluding from the model-based results to the original Rastrigin $2 \mathrm{D}$ function results

\begin{tabular}{ccccccc} 
from & \multicolumn{2}{c}{$\mathrm{m}-100$} & \multicolumn{2}{c}{$\mathrm{m}-200$} & \multicolumn{2}{c}{$\mathrm{m}-400$} \\
to & TQP & TQM & TQP & TQM & TQP & TQM \\
quality & 0.418 & 0.129 & 0.441 & 0.132 & 0.442 & 0.122 \\
budget & 0.429 & 0.300 & 0.450 & 0.296 & 0.454 & 0.288
\end{tabular}

obtained values are not very accurate. Note that this does not harm the approach as we utilize the repeats here only to assess the quality of a parameterization in comparison to the algorithm tuning on the real problem. In a real-world application, such comparison would not be possible anyway.

We also conclude that capturing the basin structure is important for testing/tuning algorithms on a model instead of the real problem. This is different from surrogate model usage within an optimization algorithm where obtaining the rough global structure is an advantage.

\section{CONCLUSIONS}

The results from our experiments on some benchmark functions support our conjecture that the concept of using a surrogate model approach for tuning optimization algorithms possesses a fruitful and as yet unexplored potential for optimization under scarce resources. Under the aspect that tuning on models built from existing problem samples comes at virtually no cost, the TQP must only be higher than $25 \%$ and the TQM below $50 \%$ to obtain a better than median parameterization without spending more precious evaluations. This is clearly possible, as demonstrated in our experiments. However, it remains to identify under which conditions the surrogate models reliably capture the main characteristics of the problem. To this edge, some more work concerning these problem properties and their interaction with algorithm parameters would be helpful. Tests with more algorithms and more problems could pave the way for improved models, finally hopefully leading to an integrated method that works as black box for the potential user who is somebody with a computationally very expensive problem.

\section{REFERENCES}

[1] Anne Auger and Nikolaus Hansen. A Restart CMA Evolution Strategy With Increasing Population Size. In B. McKay et al., editors, Proc. 2005 Congress on Evolutionary Computation (CEC'05), pages 1769-1776, Piscataway NJ, 2005. IEEE Press.

[2] Thomas Bartz-Beielstein. Experimental Research in Evolutionary Computation - The New Experimentalism. Natural Computing Series. Springer, Berlin, 2006.

[3] Thomas Bartz-Beielstein, Mike Preuß, Karlheinz Schmidt, and Hans-Paul Schwefel. Model optimization with evolutionary algorithms. In K. Lucas and P. Roosen, editors, Emergence, Analysis, and Evolution of Structures - Concepts and Strategies Across Disciplines, pages 47-62. Springer, Berlin, 2010.

[4] H.-G. Beyer and H.-P. Schwefel. Evolution strategies: A comprehensive introduction. Natural Computing, $1(1): 3-52,2002$.

[5] Dirk Biermann, Klaus Weinert, and Tobias Wagner. Model-based optimization revisited: Towards real-world processes. In IEEE Congress on
Evolutionary Computation, 2008. CEC 2008., pages 2975-2982, June 2008.

[6] M. Emmerich, K. Giannakoglou, and B. Naujoks. Single- and multi-objective evolutionary optimization assisted by gaussian random field metamodels. IEEE Transactions on Evolutionary Computation, 10(4):421-439, 2006

[7] Alexander Forrester, Andras Sobester, and Andy Keane. Engineering Design via Surrogate Modelling: A Practical Guide. Wiley, 2008.

[8] N. Hansen, A. Auger, S. Finck, and R. Ros. Real-parameter black-box optimization benchmarking 2009: Experimental setup. Technical Report RR-6828, INRIA, 2009

[9] N. Hansen and A. Ostermeier. Completely Derandomized Self-Adaptation in Evolution Strategies. IEEE Computational Intelligence Magazine, 9(2):159-195, 2001.

[10] Nikolaus Hansen. The CMA Evolution Strategy: A Tutorial, April 26 2008. accessed 21-01-09, http://www.bionik.tuberlin.de/user/niko/cmatutorial.pdf.

[11] Nikolaus Hansen and Stefan Kern. Evaluating the cma evolution strategy on multimodal test functions. In X. Yao, H.-P. Schwefel, et al., editors, Parallel Problem Solving from Nature - PPSN VIII, Proc. Eighth Int'l Conf., Birmingham, pages 282-291, Berlin, 2004. Springer.

[12] Kenneth De Jong. Parameter Setting in EAs: a 30 Year Perspective. In Fernando G. Lobo and Cláudio F. Lima and Zbigniew Michalewicz, editor, Parameter Setting in Evolutionary Algorithms. Springer, Berlin, 2007.

[13] Stefan Kern, Nikolaus Hansen, and Petros Koumoutsakos. Local meta-models for optimization using evolution strategies. In Parallel Problem Solving from Nature - PPSN IX, pages 939-948, 2006.

[14] Søren N. Lophaven, Hans B. Nielsen, and Jacob Søndergaard. DACE - A Matlab Kriging Toolbox. Technical Report IMM-REP-2002-12, Informatics and Mathematical Modelling, Technical University of Denmark, Copenhagen, Denmark, August 2002.

[15] Mike Preuss. Adaptability of algorithms for real-valued optimization. In Mario Giacobini et al., editors, Applications of Evolutionary Computing, EvoWorkshops 2009. Proceedings, volume 5484 of Lecture Notes in Computer Science, pages 665-674, Berlin, 2009. Springer.

[16] Günter Rudolph, Mike Preuss, and Jan Quadflieg. Two-layered surrogate modeling for tuning optimization metaheuristics. Technical Report TR09-2-005, Technische Universität Dortmund, Germany, Sept. 2009. http://ls11-www.cs.tudortmund.de/_media/techreports/tr09-005.pdf.

[17] Jerome Sacks, William J. Welch, Toby J. Mitchell, and Henry P. Wynn. Design and analysis of computer experiments. Statistical Science, 4(4):409-423, November 1989.

[18] Thomas J. Santner, Brian J. Williams, and William I. Notz. The Design and Analysis of Computer Experiments. Springer, 2003.

[19] P. N. Suganthan, N. Hansen, J. J. Liang, K. Deb, Y.-P. Chen, A. Auger, and S. Tiwari. Problem definitions and evaluation criteria for the cec 2005 special session on real-parameter optimization. Technical report, Nanyang Technological University, Singapore, May 2005. http://www.ntu.edu.sg/home/EPNSugan.

[20] Aimo Törn, Montaz Ali, and Sami Viitanen. Stochastic Global Optimization: Problem Classes and Solution Techniques. Journal of Global Optimization, 14(4):437-447, 1999. 